\title{
A two-component phenomenology for homogeneous magnetohydrodynamic turbulence
}

\author{
S. Oughton ${ }^{\mathrm{a})}$ \\ Department of Mathematics, University of Waikato, Private Bag 3105, Hamilton, New Zealand \\ P. Dmitruk and W. H. Matthaeus \\ Bartol Research Institute, University of Delaware, Newark, Delaware 19716
}

(Received 28 September 2005; accepted 27 February 2006; published online 21 April 2006)

\begin{abstract}
A one-point closure model for energy decay in three-dimensional magnetohydrodynamic (MHD) turbulence is developed. The model allows for influence of a large-scale magnetic field that may be of strength sufficient to induce Alfvén wave propagation effects, and takes into account components of turbulence in which either the wave-like character is negligible or is dominant. This two-component model evolves energy and characteristic length scales, and may be useful as a simple description of homogeneous MHD turbulent decay. In concert with spatial transport models, it can form the basis for approximate treatment of low-frequency plasma turbulence in a variety of solar, space, and astrophysical contexts. (C) 2006 American Institute of Physics.
\end{abstract}

[DOI: 10.1063/1.2188088]

\section{INTRODUCTION}

Although the turbulence problem for both neutral (hydrodynamic) and electrically conducting fluids is far from solved, the basic physics of turbulent energy decay for the hydrodynamic problem has been reasonably well understood, albeit approximately, for nearly 70 years. ${ }^{1-4}$ The elementary idea is that the cascade - or transfer of energy from large scales to small scales-proceeds with intensity that mainly depends upon just a few properties of the large-scale "energy-containing" eddies. For the simplest case of isotropic homogeneous turbulence, the two key quantities are the mean-square velocity of turbulent motions (turbulence energy per unit mass) $u^{2}$ and a single similarity length scale $\lambda$, which serves to define the scale of the energy-containing motions. The decay rate of turbulence energy $d u^{2} / d t \equiv-\varepsilon$ $\sim-u^{3} / \lambda$ is consistent with a family of decay models, including self-preservation of the functional form of the two-point correlation function ${ }^{2}$ or related models with specified conservation properties. ${ }^{5}$

Magnetohydrodynamics (MHD) is more complex, even in its isotropic form, in that it involves both a velocity field $\mathbf{v}$ and a magnetic field $\mathbf{b}$. Additional influences can therefore enter, such as the correlation between $\mathbf{v}$ and $\mathbf{b}$, and, in principle, two or more length scales. Nevertheless, the basic picture survives, namely that energy transfers from large scales to small scales, with the decay rate controlled by energycontaining eddies, and more or less independent of the details of the small-scale dissipation processes. Consequently, phenomenological "one-point closure" models involving just a few degrees of freedom are found to be reasonably accurate in accounting for the gross features of isotropic MHD turbulence decay in moderate Reynolds numbers simulations. ${ }^{6-8}$ One-point models have also been used as components in more elaborate transport theories to explain, for example,

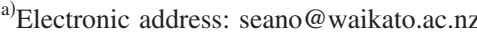

radial evolution of plasma turbulence in the solar wind. ${ }^{9}$

The situation becomes more complex when MHD turbulence is anisotropic due to a large-scale dc magnetic field. In particular, the added complication of wave propagation, strongly influencing some parts of the fluctuation spectrum while only minimally affecting other parts, makes it difficult to argue that the simplest phenomenological decay models afford an adequate description. Here we develop a more elaborate decay model consisting of two underlying components - one more wave-like and one less so- and suggest that this may better describe MHD turbulence in the presence of a large-scale magnetic field.

The essence of our approach is to consider the incompressible MHD fluctuations as being composed of two interacting ingredients, namely quasi-two-dimensional fluctuations, for which nonlinear effects dominate over wave propagation ones, and wave-like fluctuations, for which the propagation effects are important. As a shorthand, we often refer to these components as Q2D and waves, respectively.

\section{WAVES AND LOW-FREQUENCY TURBULENCE}

To facilitate a systematic construction of models for the time evolution of several relevant energy-like quantities, we first decompose the fluctuating velocity and magnetic field (in Alfvén units) as

$$
\begin{aligned}
& \mathbf{v}=\mathbf{v}^{\mathrm{Q} 2 \mathrm{D}}+\mathbf{v}^{\text {waves }}, \\
& \mathbf{b}=\mathbf{b}^{\mathrm{Q} 2 \mathrm{D}}+\mathbf{b}^{\text {waves }} .
\end{aligned}
$$

The label "Q2D" denotes fluctuations that are quasi-twodimensional in the sense to be described presently. The decomposition can be accomplished readily in wave-vector space. It will also be convenient to employ Elsässer variables for each component; we denote the quasi-2D fluctuations by $\mathbf{z}_{ \pm}=\mathbf{v}^{\mathrm{Q} 2 \mathrm{D}} \pm \mathbf{b}^{\mathrm{Q} 2 \mathrm{D}}$, and the wave-like fluctuations by $\mathbf{w}_{ \pm}$ $=\mathbf{v}^{\text {waves }} \pm \mathbf{b}^{\text {waves }}$. In view of the structure of the MHD equa- 
tions (see below) both the energy $E=\left\langle|\mathbf{v}|^{2}+|\mathbf{b}|^{2}\right\rangle / 2$ and the cross helicity $H_{c}=\langle\mathbf{v} \cdot \mathbf{b}\rangle$ content are of importance. A mean magnetic field $\mathbf{B}_{0}$ is also assumed to be present. Note that in the units employed, $B_{0} \equiv V_{A}$, the large-scale Alfvén speed.

The imposed distinction between these two components is that the quasi-2D fluctuations are those for which the nonlinear time scale is shorter than the wave time scale, as was first considered by Montgomery and Turner. ${ }^{10}$ Therefore, turbulence effects for these fluctuations proceed relatively unimpeded by wave propagation effects. This defining property becomes precise when a specific functional form for the nonlinear time is adopted, along with a specific wave dispersion relation. One can build up the quasi-2D and wave-like fluctuation fields by superposing Fourier modes that obey the appropriate time-scale inequality, according to standard estimates. For the wave time scale, and assuming incompressibility (or near incompressibility ${ }^{11}$ ), the relevant wave dispersion relation for the Alfvén mode is $\omega=\mathbf{k} \cdot \mathbf{V}_{A}$, relating frequency $\omega$ to the wave vector $\mathbf{k}$. We emphasize that there is no implication here that any particular Fourier mode is precisely described as a linear wave; rather the suggestion is that, even when nonlinear couplings are present, the Alfvénic couplings induce variations with (Alfvén) time scale $\tau_{\mathrm{A}}(\mathbf{k})$ $=\left(\mathbf{k} \cdot \mathbf{V}_{A}\right)^{-1}=\left(k_{\|} V_{A}\right)^{-1}$.

According to the usual estimate based on the structure of the MHD equations, the nonlinear time at wave number $k$ is $\tau_{\mathrm{nl}}(k)=\left(k u_{k}\right)^{-1}$. The characteristic fluctuation amplitude $u_{k}$ at wave number $k$ and the omnidirectional energy spectrum $E(k)$ are related by $u_{k}^{2}=k E(k)$. When cross-helicity effects are considered, the nonlinear times for the two Elsässer fields $\mathbf{u}^{ \pm}=\mathbf{v} \pm \mathbf{b}$ are $\tau_{\mathrm{nl}}^{ \pm}(k)=\left(k u_{k}^{\mp}\right)^{-1}$, with $\left(u_{k}^{ \pm}\right)^{2}=k E^{ \pm}(k)$ defined in terms of the omnidirectional spectra $E^{ \pm}(k)$ of the associated Elsässer fields. Although the underlying spectrum may be anisotropic when a large-scale magnetic field is present, we will use the omnidirectional (i.e., direction-averaged) energy in estimation of the nonlinear time. This maintains, for the anisotropic case, the plausible approximation that the nonlinear interactions are principally local in the magnitude of wave vector. ${ }^{12-15}$

In terms of these time scales, the condition $\tau_{\mathrm{nl}}(\mathbf{k})$ $<\tau_{\mathrm{A}}(\mathbf{k})$ serves to define the quasi-2D, or low-frequency fluctuations. ${ }^{10,16-21}$ For the $H_{c}=0$ case, this is equivalent to $k_{\|} V_{A}<k u_{k}$. Conversely, the wave-like fluctuations are defined by the opposite inequality: $k_{\|} V_{A}>k u_{k}$. As an example, in the solar wind there is observational evidence that both types of fluctuations are present. ${ }^{16,22}$

For a given distribution of excitations in wave-vector space, this decomposition scheme separates the Fourier components of general velocity and magnetic fields according to a definite prescription for which parts of the $\mathbf{k}$ space contribute to the two specified subpopulations (Fig. 1). A similar decomposition was discussed for solar wind turbulence, ${ }^{16,23}$ while simple idealized representations of this type, known as two-component "slab-2D" models, are employed in some solar wind and cosmic ray scattering analyses. ${ }^{24,22}$ Note that the type of two-component representation we discuss here is not a restriction to two pure symmetries as it is in the "slab$2 \mathrm{D}$ " models. Instead, a fully populated $3 \mathrm{D}$ spectrum is projected according to the given prescription.

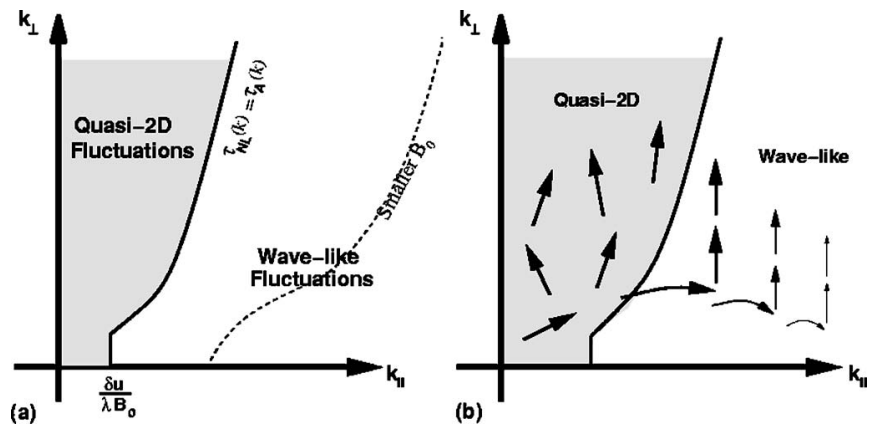

FIG. 1. (a) Sketch in Fourier space of the quasi-2D and wave-like regions, along with the boundary between them, i.e., the heavy curve labeled $\tau_{\mathrm{nl}}(\mathbf{k})=\tau_{\mathrm{A}}(\mathbf{k})$. The wave-like region is unshaded. As $B_{0}$ decreases, the equal time-scale boundary moves away from the $k_{\perp}$ axis, toward the $k_{\|}$one (e.g., dotted curve). In the $B_{0} \rightarrow 0$ limit, the shaded region occupies the entire plane, and there are no wave-like modes. (b) Schematic indication of the direction and strength of spectral transfer at selected points in Fourier space. Note the dominant perpendicular transfer in the wave-like region.

In particular, low-compressibility fluctuations of a given Elsässer type and at a given wave vector $\mathbf{k}$ are either of the low-frequency type or of the wave-like types. Thus, carrying out this projection and then assembling an (arbitrary) wavelike field $\mathbf{w}_{+}$and Q2D field $\mathbf{z}_{+}$, one finds that these are orthogonal in the sense that (at each time)

$$
\left\langle\mathbf{z}_{ \pm} \cdot \mathbf{w}_{ \pm}\right\rangle=0,
$$

where the ensemble averaging procedure $\langle\ldots\rangle$ is equivalent to a spatial average for the homogeneous turbulence case considered herein.

\section{DEVELOPMENT}

The starting point for the present development is (dissipative) incompressible MHD, with a mean magnetic field $\mathbf{B}_{0}=B_{0} \hat{\mathbf{e}}_{3}$. Substituting the decomposed velocity and magnetic fields of Eqs. (1) and (2) into the MHD equations, and switching to Elsässer variables for each component, defined as $\mathbf{z}_{ \pm}=\mathbf{v}^{\mathrm{Q} 2 \mathrm{D}} \pm \mathbf{b}^{\mathrm{Q} 2 \mathrm{D}}$ and $\mathbf{w}_{ \pm}=\mathbf{v}^{\text {waves }} \pm \mathbf{b}^{\text {waves }}$, one finds for the "plus" fields,

$$
\begin{aligned}
\frac{\partial \mathbf{z}_{+}}{\partial t}+\frac{\partial \mathbf{w}_{+}}{\partial t}= & -\mathbf{z}_{-} \cdot \nabla \mathbf{z}_{+}-\mathbf{z}_{-} \cdot \nabla \mathbf{w}_{+}-\mathbf{w}_{-} \cdot \nabla \mathbf{z}_{+}-\mathbf{w}_{-} \cdot \nabla \mathbf{w}_{+} \\
& +\mathbf{B}_{0} \cdot \nabla \mathbf{z}_{+}+\mathbf{B}_{0} \cdot \nabla \mathbf{w}_{+}-\nabla p,
\end{aligned}
$$

with a similar equation for the evolution of the "minus" fields. The $\mathbf{z}_{ \pm}$and $\mathbf{w}_{ \pm}$are solenoidal, and this constraint determines $p$, the total (mechanical plus magnetic) pressure, through a Poisson equation that results from the divergence of Eq. (4). An advantage of the two-component decomposition is that the physical nature of the nonlinear terms in Eq. (4) is evident: reading from left to right, there are terms involving Q2D-Q2D interactions, Q2D-wave, wave-Q2D, and wave-wave interactions. These are followed by propagation-dominated terms and the pressure gradient.

Further structure is revealed by forming the equations for evolution of the "plus" Elsässer energies, obtained by taking dot products of Eq. (4) with $\mathbf{z}_{+}$or $\mathbf{w}_{+}$, and then spatially averaging. After use of the orthogonality property Eq. (3), this yields for the Q2D and wave-like energies, 


$$
\begin{aligned}
\frac{1}{2} \frac{d\left\langle z_{+}^{2}\right\rangle}{d t} \sim- & \left\langle\mathbf{z}_{-} \cdot \nabla \frac{z_{+}^{2}}{2}\right\rangle-\left\langle\mathbf{z}_{+} \cdot\left(\mathbf{z}_{-} \cdot \nabla\right) \mathbf{w}_{+}\right\rangle \\
& -\left\langle\mathbf{w}_{-} \cdot \nabla \frac{z_{+}^{2}}{2}\right\rangle-\left\langle\mathbf{z}_{+} \cdot\left(\mathbf{w}_{-} \cdot \nabla\right) \mathbf{w}_{+}\right\rangle, \\
\frac{1}{2} \frac{d\left\langle w_{+}^{2}\right\rangle}{d t} \sim- & \left\langle\mathbf{z}_{-} \cdot \nabla \frac{w_{+}^{2}}{2}\right\rangle-\left\langle\mathbf{w}_{+} \cdot\left(\mathbf{z}_{-} \cdot \nabla\right) \mathbf{z}_{+}\right\rangle \\
& -\left\langle\mathbf{w}_{-} \cdot \nabla \frac{w_{+}^{2}}{2}\right\rangle-\left\langle\mathbf{w}_{+} \cdot\left(\mathbf{w}_{-} \cdot \nabla\right) \mathbf{z}_{+}\right\rangle,
\end{aligned}
$$

where the propagation and pressure terms are not written. Similar forms for the other Elsässer energies are readily derived.

Before turning to specific approximations, we note that the above two equations are really statements of conservation laws; indeed, each term on the right-hand side is either zero (those with an explicit $\frac{1}{2}$ ) or paired with a term in the "other" equation for which their sum gives zero. For example, $\left\langle\mathbf{z}_{+} \cdot\left(\mathbf{z}_{-} \cdot \nabla\right) \mathbf{w}_{+}+\mathbf{w}_{+} \cdot\left(\mathbf{z}_{-} \cdot \nabla\right) \mathbf{z}_{+}\right\rangle=\left\langle z_{-}^{\beta}\left(z_{+}^{\alpha} \partial_{\beta} w_{+}^{\alpha}+w_{+}^{\alpha} \partial_{\beta} z_{+}^{\alpha}\right)\right\rangle$ $=\left\langle\nabla \cdot\left[\mathbf{z}_{-}\left(\mathbf{z}_{+} \cdot \mathbf{w}_{+}\right)\right]\right\rangle=0$.

Despite these conservation properties, the structure of the terms can still be used to advantage in modeling several nonlinear cascade effects. The shortcut employed affords substantial algebraic savings and is based on writing $0=a$ $+(-a)$. Focusing on a particular term as an illustration, one readily sees that the first contribution to Eq. (5) is

$$
\begin{aligned}
\left.\frac{d\left\langle z_{+}^{2}\right\rangle}{d t}\right|_{\mathbf{z}_{-} \cdot \nabla \mathbf{z}_{+}} & =-2\left\langle\mathbf{z}_{+} \cdot\left(\mathbf{z}_{-} \cdot \nabla\right) \mathbf{z}_{+}\right\rangle \\
& \propto \sum_{\mathbf{k}} \widetilde{\mathbf{z}}_{+}(-\mathbf{k}) \cdot \sum_{\mathbf{p}+\mathbf{q}=\mathbf{k}} \mathbf{q} \cdot \widetilde{\mathbf{z}}_{-}(\mathbf{p}) \widetilde{\mathbf{z}}_{+}(\mathbf{q}) \\
& =2 \sum_{\mathbf{k}} T(\mathbf{k})=0,
\end{aligned}
$$

where $\widetilde{\mathbf{z}}(\mathbf{k})$ are the Fourier components of $\mathbf{z}(\mathbf{x})$, and $\mathbf{k}, \mathbf{p}$, and $\mathbf{q}$ are wave vectors. The energy transferred into mode $\mathbf{k}$ by the (selected) nonlinear couplings is $T(\mathbf{k})$ (in general this would include contributions from all relevant nonlinear terms). It is easy to show, in either real or wave-vector space, that these terms vanish, for suitable boundary conditions. This represents the fact that the nonlinear couplings in question rearrange energy in $\mathbf{k}$ space but do so without changing the total. Energy decay occurs because the dissipation term (e.g., viscosity, not written above) engages at small scales and removes energy from the system. The nonlinear terms, in a statistical sense, spread energy over all scales; this leads to a replenishing of the energy lost at small scales (to dissipation), thereby establishing the cascade. Thus, significance is attached to rewriting $\Sigma_{\mathbf{k}} T(\mathbf{k})=0$ as

$$
-\sum_{\text {low } \mathbf{k}} T(\mathbf{k})=\sum_{\text {high } \mathbf{k}} T(\mathbf{k})=\text { rate of dissipation, }
$$

with some appropriate choice of cutoff between small and large wave numbers implied. Our approach is to model the left hand side of this equation using the structural form of each nonlinear term, evaluated using a suitably chosen ("energy-containing") scale that characterizes the source of the energy being transferred to small scales.

For this first presentation of the model, we specialize to the case of zero cross helicity for each component, e.g., $\left\langle\mathbf{v}^{\mathrm{Q} 2 \mathrm{D}} \cdot \mathbf{b}^{\mathrm{Q} 2 \mathrm{D}}\right\rangle=0$. It is then convenient to employ the notation

$$
\begin{aligned}
& Z^{2}=\left\langle z_{+}^{2}\right\rangle=\left\langle z_{-}^{2}\right\rangle, \\
& W^{2}=\left\langle w_{+}^{2}\right\rangle=\left\langle w_{-}^{2}\right\rangle,
\end{aligned}
$$

so that the conventional factor of one-half appears in both representations of the total fluctuation energy, $E=\frac{1}{2}\left\langle v^{2}+b^{2}\right\rangle$ $=\frac{1}{2}\left(Z^{2}+W^{2}\right)$.

\section{A. Modeling nonlinear terms}

Up to this point, everything is essentially exact. The next step is to model each of the terms on the RHS of Eqs. (5) and (6). We base the modeling on the well-established von Kármán-Taylor ${ }^{2,3}$ approach for energy decay phenomenology in hydrodynamics, that is, on the pair of equations $d u^{2} / d t \sim-u^{2} / \tau_{\mathrm{sp}}$ and $d \ell / d t \sim \ell / \tau_{\mathrm{sp}}$, with the spectral transfer time $\tau_{\mathrm{sp}}$ identified with the global hydrodynamic nonlinear (or "eddy turnover") time $\tau_{\mathrm{nl}}=\ell / u$. Analogous equations for $\mathrm{MHD}^{12,25,26,6}$ have been written as direct extensions of this isotropic homogeneous hydrodynamic case, and these have been found to account well for decaying MHD turbulence simulations. ${ }^{6,27,28}$ Here, we will adopt a straightforward generalization to account for two evolving MHD components.

\section{General approach}

The nonlinear terms in the energy evolution equations are of the form $\left\langle\mathbf{A}_{3} \cdot\left(\mathbf{A}_{1} \cdot \nabla\right) \mathbf{A}_{2}\right\rangle$. We model these with approximations $\sim A_{3} A_{2} / \tau_{\text {sp }}$ for amplitudes $A_{j}(=Z$ or $W)$ and an appropriate spectral transfer time $\tau_{\mathrm{sp}}$. In constructing the $\tau_{\mathrm{sp}}$, we use the "golden rule" ${ }^{25} \tau_{\mathrm{sp}}=\tau_{\mathrm{nl}}^{2}$, where $\tau_{\mathrm{nl}}$ is the estimated nonlinear time scale for the coupling in question, and $\tau_{3}$ is the estimated triple decay time for the (cubic) correlation associated with this coupling. ${ }^{12,25,26,29,30}$ Generally the triple decay rate is the sum of (all) uncorrelated rates ${ }^{30}$ that limit the lifetime of this nonlinear term..$^{31,12,32,33}$ Typically, $1 / \tau_{3}=1 / \tau_{\mathrm{nl}}+1 / \tau_{\mathrm{A}}+\ldots$, where contribution is anticipated from the nonlinear time and the appropriate Alfvén time scale $\tau_{\mathrm{A}}$. Indeed, if these are the only contributors to $\tau_{3}$, one obtains

$$
\tau_{3}=\frac{\tau_{\mathrm{nl}}}{1+\tau_{\mathrm{nl}} / \tau_{\mathrm{A}}},
$$

a result used repeatedly below. Note that the nonlinear time associated with $\mathbf{A}_{1} \cdot \nabla \mathbf{A}_{2}$ is $\tau_{\mathrm{nl}}=L_{A 2} / A_{1}$, with $L_{A 2}$ the energycontaining length scale associated with field $\mathbf{A}_{2}$. Hereafter, we refer to those terms in Eqs. (5) and (6) which have an explicit factor of $\frac{1}{2}$ as cascade terms, while the others are called exchange terms. The distinction between these is in some ways just semantic since all the terms have the same structural form, namely $\left\langle\mathbf{A}_{3} \cdot\left(\mathbf{A}_{1} \cdot \nabla\right) \mathbf{A}_{2}\right\rangle$. Nonetheless, it provides a useful organizing framework and the physics is distinct depending upon whether $\mathbf{A}_{3}$ and $\mathbf{A}_{2}$ are the same field. When they are, excitation is transferred within that same 
field/component (i.e., a cascade-type term). In the other case- $\mathbf{A}_{3}$ and $\mathbf{A}_{2}$ distinct fields - excitation can be moved between components. We now discuss some representative examples for each of these types.

\section{Cascade terms}

In this subsection, we model the two cascade terms in the $W^{2}$ energy equation. As will be seen, one of these terms involves resonant interactions while the other does not. Consider first $\left\langle\mathbf{w}_{-} \cdot \nabla \mathbf{w}_{+}^{2} / 2\right\rangle=\left\langle\mathbf{w}_{+} \cdot\left(\mathbf{w}_{-} \cdot \nabla\right) \mathbf{w}_{+}\right\rangle$in Eq. (6). The interpretation is clear: the $\mathbf{w}_{-}$field strains the $\mathbf{w}_{+}$field, causing a transfer of spectral excitation that projects back onto the $\mathbf{w}_{+}$ field, although at a different wave vector [cf. the structure of the convolution sum in Eq. (7)]. As explained in Sec. III A 1, this transfer is modeled in the form $W^{2} / \tau_{\mathrm{sp}}$, with estimates for $\tau_{\mathrm{nl}}$ and $\tau_{3}$ needed in the construction of $\tau_{\mathrm{sp}}$. In this case, the appropriate nonlinear time is $\tau_{\mathrm{nl}}^{w W}=\lambda / W$, where the speed used is associated with the field affecting the straining motion $(W)$, and the gradient is approximated using $\lambda$, the similarity scale or energy-containing scale of the field being strained (also $W$ ).

To compute the triple decay time, we need to include the effects of $\tau_{\mathrm{nl}}^{w w}$ and also those of Alfvén wave propagation, in this case controlled by the wave period of the $W$ fluctuations, $\tau_{\mathrm{A}}=\lambda_{\|} / V_{A}$. Using the expression (11) for $\tau_{3}$ and applying the golden rule then yields

$$
\frac{1}{\tau_{\mathrm{sp}}}=\frac{\tau_{3}}{\left(\tau_{\mathrm{nl}}^{w w}\right)^{2}}=\frac{W}{\lambda} \frac{W / \lambda}{W / \lambda+V_{A} / \lambda_{\|}},
$$

where $\lambda_{\|}$is a typical parallel length scale for the $W$ fluctuations. Thus, the sought after approximation for this nonlinear cascade term is

$$
\left.\frac{1}{2} \frac{d W^{2}}{d t}\right|_{\mathbf{w}_{-} \cdot \nabla w_{+}^{2} / 2} \approx-\frac{W^{3}}{\lambda}\left[\frac{W / \lambda}{W / \lambda+V_{A} / \lambda_{\|}}\right],
$$

where the minus sign is implied by the identification of this term as one that drives the cascade to higher wave number where dissipation removes energy. Such wave-wave interactions can be identified with spectral transfer of the Iroshnikov-Kraichnan type. ${ }^{34,31}$ Since these couplings involve the interaction of counterpropagating modes, they are nonresonant.

Turning now to the other cascade term in Eq. (6), namely $\left\langle\mathbf{z}_{-} \cdot \nabla \mathbf{w}_{+}^{2} / 2\right\rangle$, the approach outlined above indicates that this too will be modeled as $W^{2} / \tau_{\mathrm{sp}}$. However, in this case $\mathbf{w}_{+}$is strained by $\mathbf{z}_{-}$. While this presents no problem in constructing the appropriate nonlinear time $\tau_{\mathrm{nl}}^{z w}=\lambda / Z$, construction of the triple correlation time is more subtle. (The superscripts on $\tau_{\mathrm{nl}}^{z w}$ are ordered so as to match the order of the fields in the nonlinear term being modeled, here $\mathbf{z}_{-} \cdot \nabla \mathbf{w}_{+}$.)

Both $\mathbf{z}_{-}$and $\mathbf{w}_{+}$have oscillation periods associated with them, respectively $\tau_{\mathrm{A}}^{z} \approx \ell_{\|} / V_{A}$ and $\tau_{\mathrm{A}}^{w} \approx \lambda_{\|} / V_{A}$, where $\ell_{\|}$is a characteristic parallel length scale for the Q2D component. Since the latter time scale is typically (much) shorter than the former, it might be thought that it would dominate in $\tau_{3}$. (Here, we have in mind that the bulk of $W$ energy will typically reside in parallel scales considerably shorter than those characteristic of $Z$. Situations where $\lambda_{\|}$is only a little shorter than $\ell_{\|}$can be envisaged.) To see why this is not so, consider an arbitrary triad belonging to this class of couplings. Because the triad is a $Z$ interacting with a $W$ to drive another $W$, the driven mode will typically have approximately the same parallel wave number as the driving $W$ mode. Thus, as far as oscillation is concerned, these two $W$ modes are phase locked-or in (near) resonance-and their oscillation time scale is irrelevant. That leaves $\tau_{\mathrm{A}}^{z}$ to contribute to $\tau_{3}$, along with $\tau_{\mathrm{nl}}^{z w}$. Employing again Eq. (11) and the golden rule, we obtain

$$
\frac{1}{\tau_{\mathrm{sp}}}=\frac{\tau_{3}}{\left(\tau_{\mathrm{nl}}^{z w}\right)^{2}}=\frac{Z}{\lambda} \frac{Z / \lambda}{Z / \lambda+V_{A} / \ell_{\|}},
$$

which can be substituted into $-W^{2} / \tau_{\mathrm{sp}}$ to give the final modeled form for $\left\langle\mathbf{z}_{-} \cdot \nabla \mathbf{w}_{+}^{2} / 2\right\rangle$.

Modeling of the cascade terms in the $Z$ energy equation proceeds similarly. Note that in that equation both terms are of the resonant type.

\section{Exchange terms}

Among the nonlinear terms on the right hand sides of Eqs. (5) and (6) are several that cannot be easily written as a flux of an energy density, and which therefore are not readily interpreted as direct cascade terms in the sense of the previous subsection.

These terms occur in pairs, with one member in Eq. (5) and the other in Eq. (6). For example, $\left\langle\mathbf{z}_{+} \cdot\left(\mathbf{z}_{-} \cdot \nabla\right) \mathbf{w}_{+}\right\rangle$and $\left\langle\mathbf{w}_{+} \cdot\left(\mathbf{z}_{-} \cdot \nabla\right) \mathbf{z}_{+}\right\rangle$is one such pair, which we consider in detail below. (The other pair of exchange terms is modeled in analogous fashion.) Moreover the changes of energy in each pair sum to zero, as shown below Eq. (6). Hence these couplings can be viewed as conservative exchanges between the $W$ and $Z$ classes of excitation.

Following the ideas noted above, we adopt the form $W Z / \tau_{\mathrm{sp}}$ for contributions originating with these terms. For the effect on the $Z$ energy, Eq. (5), of the first listed term in the above example, we estimate an exchange with magnitude

$$
\left\langle\mathbf{z}_{+} \cdot\left(\mathbf{z}_{-} \cdot \nabla\right) \mathbf{w}_{+}\right\rangle \rightarrow Z W \frac{\tau_{3}}{\left(\tau_{\mathrm{nl}}^{z W}\right)^{2}}=\frac{W Z^{2}}{\lambda}\left[\frac{Z / \lambda}{Z / \lambda+V_{A} / \lambda_{\|}}\right] .
$$

Note that the nonlinear time is based on an energycontaining length, $\lambda$, for the wave-like field, but a Q2D speed, $Z$. The Alfvén time (contributing to the $\tau_{3}$ ) is estimated as $\tau_{\mathrm{A}}=\lambda_{\|} / V_{A}$ and based only on $W$ quantities. (A refinement to the model can be made here, with the $\lambda_{\|}$appearing in the two $\tau_{\mathrm{A}}$ for this pair of exchange terms being replaced with $\ell_{\|} / 2$. The motivation is that the latter value is of order the minimum energy-containing scale that could arise from the interaction of two $Z$ modes.) This is because the straining (by $\mathbf{z}_{-}$) is always nonresonant with the $\mathbf{w}_{+}$field with which it is interacting, to pump $Z$. We assign this estimate as a gain of Q2D energy.

In the complementary (energy-conserving) term in Eq. (6), there is an analogous contribution that we estimate accordingly as 


$$
\left\langle\mathbf{w}_{+} \cdot\left(\mathbf{z}_{-} \cdot \nabla\right) \mathbf{z}_{+}\right\rangle \rightarrow W Z \frac{\tau_{3}}{\left(\tau_{\mathrm{nl}}^{z^{2}}\right)^{2}}=\frac{W Z^{2}}{\ell}\left[\frac{Z / \ell}{Z / \ell+V_{A} / \lambda_{\|}}\right] .
$$

In this case, the length scale obtained from the gradient is the Q2D one $\ell$, whereas the $\tau_{\mathrm{A}}$ is unchanged. This term is associated with a gain of $W$ energy. [Actually, the LHSs of Eqs. (15) and (16) are equal and opposite and, ideally, therefore so should their modeled versions be. However, they differ in the way the length scale enters in the respective $\tau_{\mathrm{nl}}$, so that we reinstate conservation by hand-inserting the negative of each modeled term in the partner equation.]

Finally, since the exchanges are modeled as conservative, the negative of Eq. (15) appears in the $W^{2}$ equation, while the negative of Eq. (16) will appear in the $Z^{2}$ equation.

\section{B. Two-component phenomenology \\ 1. Evolution of the energies}

When the above reasoning is applied successively to each nonlinear coupling in Eqs. (5) and (6), one obtains

$$
\begin{aligned}
\frac{1}{2} \frac{d Z^{2}}{d t}= & -\frac{Z^{3}}{\ell} \Gamma_{z}^{z z}-\frac{W Z^{2}}{\ell} \Gamma_{z}^{w z}+W Z^{2}\left[\frac{\Gamma_{w}^{z w}}{\lambda}-\frac{\Gamma_{w}^{z z}}{\ell}\right] \\
& +W^{2} Z\left[\frac{\Gamma_{w}^{w w}}{\lambda}-\frac{\Gamma_{w}^{w z}}{\ell}\right], \\
\frac{1}{2} \frac{d W^{2}}{d t}= & -\frac{W^{2} Z}{\lambda} \Gamma_{z}^{z w}-\frac{W^{3}}{\lambda} \Gamma_{w}^{w w}-W Z^{2}\left[\frac{\Gamma_{w}^{z w}}{\lambda}-\frac{\Gamma_{w}^{z z}}{\ell}\right] \\
& -W^{2} Z\left[\frac{\Gamma_{w}^{w w}}{\lambda}-\frac{\Gamma_{w}^{w z}}{\ell}\right],
\end{aligned}
$$

where for compactness the attenuation factors associated with the triple decay rate are written as

$$
\Gamma_{c}^{a b}=\frac{1}{1+\tau_{\mathrm{nl}}^{a b} / \tau_{\mathrm{A}}^{c}}=\frac{a / L_{b}}{a / L_{b}+V_{A} / L_{c}^{\|}} .
$$

Here, $a, b$, and $c$ represent the appropriate $\mathbf{z}_{ \pm}, \mathbf{w}_{ \pm}$fluctuation, $L_{b}$ is a characteristic length scale of $b$, and $L_{c}^{\|}$is a characteristic parallel length scale of $c$. Length scales associated with the quasi-2D component are denoted using $\ell$, while those for the wave-like component are denoted with $\lambda$.

Resonant terms can be identified by the presence of a $z$ subscript (rather than a $w$ ) on the $\Gamma$ factors and are only weakly dependent on $B_{0}$. In Eq. (17) for the $Z$ energy, the first term corresponds to ZZZ interactions which are always (otherwise known as "trivially") resonant. The second term in this equation is also (quasi)resonant, although more weakly since it models a $Z$ and a $W$ mode interacting to drive another $Z$. For the $W$ energy, only the first term of Eq. (18) is resonant, corresponding to the process in which a wave-like fluctuation interacts with a Q2D one to drive another wavelike one. This process was first identified by Shebalin et al. ${ }^{35}$

Note that for computational purposes, it is useful to rewrite the LHS of Eq. (17) as $Z d Z / d t$, and then divide through by $Z$. This makes no difference when $Z \neq 0$, but does allow $Z$ to grow from a zero value, which is impossible when Eq. (17) is used directly. Similar comments apply to the $W^{2}$ equation.

\section{Evolution of the length scales}

In order to close the model, the length scales $-\ell, \lambda, \ell_{\|}$, and $\lambda_{\|}$-are also required. Specified constant length scales ${ }^{1}$ typically are not sufficient to properly capture the dynamics $^{2,27}$ and a dynamical formulation is preferable. For freely decaying turbulence, hydrodynamic antecedents suggest that a satisfactory way to proceed is to specify a conservation law that relates the evolution of the energy to the evolution of the corresponding similarity length scale.

Here, we suggest the following perhaps simplest generalization of previous MHD length-scale formulations. For the similarity scales $\ell$ and $\lambda$, we use separate conservation laws for the quasi-2D and wave-like components:

$$
\begin{aligned}
& \ell Z^{n}=\text { const, } \\
& \lambda W^{m}=\text { const, }
\end{aligned}
$$

with $n$ and $m$ independent constants. Specific choices of $n$ or $m$ can be associated with physical assumptions. ${ }^{27}$ For example, $n$ or $m=1$ implies evolution at constant Reynolds number, while $n$ or $m=2$ implies constant area under the associated correlation function.

For freely decaying turbulence, the conservation law may be used to directly compute the evolution of the length scales, e.g., $\ell(t)=Z^{n}(0) \ell(0) / Z^{n}(t)$, given initial values. Alternatively, differential equations for the time evolution of $\ell$ and $\lambda$ are obtained by differentiating Eqs. (20) with respect to time and using Eqs. (17) and (18). Note that these formulations are generally consistent with the expectation that $d L / d t \propto L / \tau_{\mathrm{sp}}$, where $L$ is the similarity scale and $\tau_{\mathrm{sp}}$, as above, is an appropriately constructed spectral transfer time.

The dynamical behavior of the wave-like parallel length scale, $\lambda_{\|}$, is somewhat more subtle, because the presence of the large-scale magnetic field $\mathbf{B}_{0}$ induces an anisotropy in the spectral transfer that suppresses parallel transfer. ${ }^{35,36}$ One would expect that this suppression would also diminish the dynamical changes to the parallel length scales. This suggests that in constructing a model to evolve the parallel length scales, one should not use the full spectral transfer rate. Our proposal here is to employ a spectral transfer rate that includes only contributions that are suppressed with increasing $B_{0}$ (cf. Refs. 6, 27, and 37). Thus, the wave-like parallel scale is reasonably modeled as evolving according to

$$
\frac{d \lambda_{\|}}{d t}=\left[\frac{W}{\lambda} \Gamma_{w}^{w w}\right] \lambda_{\|},
$$

where the factor in square brackets is an estimate of the appropriate spectral transfer rate retaining only the factor associated with the second (nonresonant) term in Eq. (18).

Similar comments apply to the Q2D parallel length scale, $\ell_{\|}$. However, in a sense, this length scale can also be taken to be defined by the (global) equal time-scale condition for the $Z$ modes: $\ell / Z=\ell_{\|} / V_{A}$. Here we employ such a definition for it, 


$$
\ell_{\|}= \begin{cases}\ell, & Z \geqslant V_{A} \\ \frac{V_{A}}{Z} \ell, & Z<V_{A} .\end{cases}
$$

Thus, if the mean field is weak, there is no distinction between $\ell$ and $\ell_{\|}$, as is suitable for the approximate isotropy expected. On the other hand, $\ell_{\|}$gets larger as the (relative) mean field strength increases. Fortunately, the parallel length scales will evolve slowly in most circumstances, so the overall results should not be very sensitive to this aspect of the phenomenology.

Equations (17) to (22) comprise a closed phenomenology for the evolution of the energies and characteristic length scales of the wave-like and Q2D components of a general MHD system. In the following sections, we discuss their form in several limits, and present a numerical solution by way of example.

\section{Limits}

The goal of the two-component model is to approximately describe the influence of an externally controlled or very large-scale mean magnetic field on the dynamics of energy decay in MHD. Therefore, it is of particular interest to examine the limiting cases of weak and strong mean magnetic field: $B_{0} \rightarrow 0$ and $B_{0} \rightarrow \infty$.

In the weak mean field case $\mathbf{B}_{0} \rightarrow 0$ (and $V_{A} \rightarrow 0$ ), and all the $\Gamma$ factors approach unity. Moreover, for consistency of interpretation it is also necessary that $W^{2} / Z^{2} \rightarrow 0$. This can be understood heuristically with reference to Fig. 1(a). As $B_{0}$ decreases, the equal time scale ${ }^{10}$ (or "critical balance" ${ }^{\text {"18) }}$ boundary expands away from the $k_{\perp}$ axis and the wave-like region occupies a smaller and smaller region of $\mathbf{k}$ space. Indeed, in the $B_{0} \rightarrow 0$ limit there is no wave-like region. Thus, only non-wave-like fluctuations remain (the terminology "quasi-2D" is misleading in this limit) and one recovers essentially the standard isotropic hydrodynamic $u^{3} / \ell$ phenomenology,

$$
\frac{1}{2} \frac{d Z^{2}}{d t}=-\frac{Z^{3}}{\ell}, \quad \frac{d \ell}{d t}=n Z .
$$

This is the sense in which the free decay of zero cross helicity MHD turbulence, without a mean field, is "hydrolike." Note that isotropy has been recovered. Analytic solutions are well-known, e.g., Refs. 3, 6, and 27, with $Z^{2}$ $\sim t^{-2 /(n+1)}$ and $\ell \sim t^{n /(n+1)}$ at large times $\left(t \gg \ell_{0} / Z_{0}\right)$.

In the strong mean field limit $\mathbf{B}_{0} \rightarrow \infty$, and the asymptotic forms of the energy decay equations are

$$
\begin{aligned}
& \frac{1}{2} \frac{d Z^{2}}{d t}=-\frac{1}{2} \frac{Z^{3}}{\ell}-\frac{W Z^{2}}{\ell} \frac{1}{1+Z / W}+\gamma \frac{\lambda_{\|}}{V_{A}}, \\
& \frac{1}{2} \frac{d W^{2}}{d t}=-\frac{Z W^{2}}{\lambda} \frac{1}{1+\lambda / \ell}-\frac{W^{4}}{\lambda^{2}} \frac{\lambda_{\|}}{V_{A}}-\gamma \frac{\lambda_{\|}}{V_{A}},
\end{aligned}
$$

where $\gamma=W Z\left(W^{2}+Z^{2}\right)\left(\ell^{2}-\lambda^{2}\right) / \ell^{2} \lambda^{2}$. In examining this limit, we retain the zeroth-order (resonant) terms, as well as the largest terms proportional to $1 / V_{A}$, in order to elucidate the

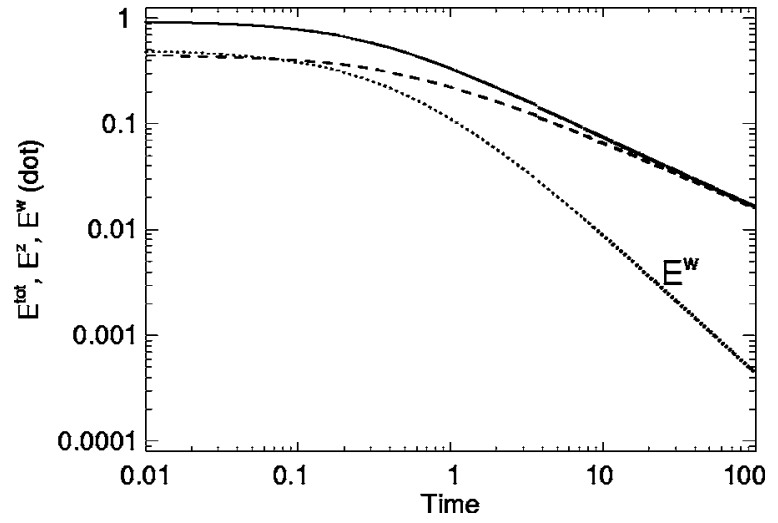

FIG. 2. Sample numerical solutions for the energies in the two-component model, Eqs. (17) to (22). The initial condition is chosen to have $W$ slightly greater than $Z$, and $\ell=1.0, \lambda=0.5$. Note the power-law evolution at larger times.

leading-order transfer of wave-like energy in cases where $Z^{2}$ is zero or very small, e.g., $Z / W \propto W / V_{A}$.

The leading-order terms for both $Z$ and $W$ are associated with (approximately) perpendicular cascades. They have the important property that they are independent of the mean field strength $B_{0}\left(\equiv V_{A}\right)$. For the wave-like component, the leading-order term is due to the dominance of the resonant interaction of a quasi-2D fluctuation with a wave-like fluctuation. The importance of these resonant terms was first identified by Shebalin et al. $^{35}$ and has been expanded upon subsequently. ${ }^{38,36,20,17}$

The leading-order Q2D behavior has two contributions. The first is again hydrodynamics-like, but with the overall strength of the decay diminished by a factor of 2 relative to the $B_{0} \rightarrow 0$ case. The second contribution also models a cascade, but a less local one. This follows since it arises from the interaction of $Z$ modes with $W$ modes and hence involves relatively large "cross channel" changes in the parallel wave numbers of the Q2D modes.

Finally, the two terms proportional to $\gamma$ are the (net) asymptotic exchange terms. They can be positive or negative depending upon which of $\ell$ and $\lambda$ is the larger.

\section{NUMERICAL ILLUSTRATION}

When investigating solutions to the two-component phenomenology developed above, there is a wide range of initial values to explore. Here we discuss an example solution for a strong mean field case, obtained using a standard fourthorder Runge-Kutta package.

Figure 2 displays the evolution of the total, Q2D, and wave-like energies, for an initial state with $Z^{2}=0.9, W^{2}=1$, and an Alfvén speed $V_{A}=20$. It can be seen that for both components the asymptotic behavior is power-law decay in time, with the Q2D component strongly dominant. The slope of the power laws depends upon the indices chosen in the conservation laws of Eqs. (20); here $n=2, m=1$. Since at later times $W \ll Z$, the asymptotic solution for the Q2D energy is $Z^{2} \sim t^{-2 /(n+1)} \sim t^{-2 / 3}$. For further discussion of decay laws, see, for example, Biskamp and Muller ${ }^{7}$ for the 3D case and Biskamp and Schwarz ${ }^{39}$ for the 2D case. 

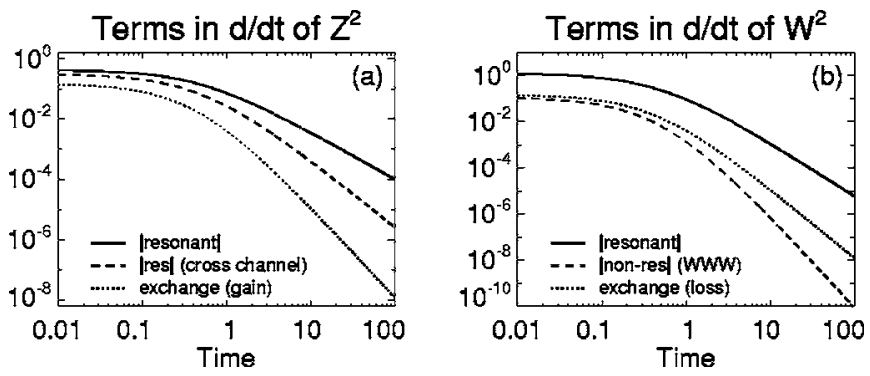

FIG. 3. Contributions of the resonant, nonresonant, and exchange terms in the equations for the energy in the (a) Q2D and (b) wave-like components. Note that for $W^{2}$, the exchange term, which is a loss of $W^{2}$ in this example, is mostly much larger than the nonresonant cascade term.

The nature of the energy decay, in tandem with the conservation laws, requires that the length scales (not shown) all tend to increasing power laws-with the exception of $\lambda_{\|}$, which is essentially constant under the influence of such a strong mean field.

It is also of interest to examine the relative contributions of the individual terms in the energy equations. A priori, one might expect the resonant terms to always be dominant. As Fig. 3 indicates, this is indeed the case for this example. Interestingly, however, the exchange term, which here is a gain for $Z^{2}$ and a loss for $W^{2}$, is always larger in magnitude than the nonresonant cascade term. Moreover, we have found other examples where the exchange term is comparable to, or even exceeds, the resonant term. (This can be achieved by increasing the initial ratio of $W / Z$ or $\ell / \lambda$, for example.) Clearly, the exchange terms can play important dynamical roles in this model for the evolution of MHD turbulence.

\section{SUMMARY AND DISCUSSION}

In a number of astrophysical, space, and solar applications, it is advantageous to treat MHD turbulence in a simple fashion, using a simple model or closure to account for the effects of turbulent cascade induced by nonlinear couplings. Due to the anisotropy induced by a large-scale regular magnetic field, low compressibility turbulence may require more than one simple estimate to account for its couplings. In some cases, turbulence effects are associated mainly with low-frequency fluctuations, in which Alfvén propagation effects are weak. In other cases, propagation effects may be strong, as in the "weak turbulence" regime examined by Galtier et al. ${ }^{40,41}$ Somewhat more generally, both effects may be included by modeling the turbulence as consisting of two components, which we have described as quasi-2D fluctuations, and waves.

The one-point closure, or phenomenology, that we developed here consists of self- and cross-interactions of the Q2D and wave-like ingredients. For the special case of zero cross helicity, we have offered Eqs. (17) and (18) as a basic model for these couplings, supplemented by conservation laws that provide for evolution of the requisite length scales. The structure of the model, written in words, is

$$
\begin{aligned}
\frac{d Z^{2}}{d t}= & - \text { resonant Q2D cascade }\left(\text { Kolomogorov-like }^{42}\right) \\
& + \text { non-res. replenishment from waves } \\
& - \text { non-res. loss to waves, } \\
\frac{d W^{2}}{d t}= & \left.- \text { resonant } Z-W \perp \text { cascade (Shebalin-like }{ }^{35}\right) \\
& - \text { non-res. } W-W \text { cascade }\left(\text { Kraichnan-like }^{34,31}\right) \\
& + \text { non-res. gain from Q2D } \\
& - \text { non-res. loss to Q2D, }
\end{aligned}
$$

where the phrases in parentheses indicate a name typically associated with cascades of that nature. The resonant terms are strong for all mean field strengths, whereas the nonresonant ones are proportional to $1 / \mathrm{V}_{A}$ when $V_{A}$ is large.

Although there is no real spectral information in this phenomenology, the modeling nonetheless provides some clarification of the distinction between situations when Kolmogorov-like, ${ }^{42}$ Shebalin-like, ${ }^{35}$ or IroshnikovKraichnan-type ${ }^{34,31}$ cascades might occur. For example, Kolmogorov cascades will dominate the energy decay process when $Z \gg W$. This is necessarily the case if $V_{A} \ll Z$, but can also occur when $V_{A}$ is large. On the other hand, if $Z \ll W$, at least two possibilities exist. First, if $Z / W>W / V_{A}$, then the Shebalin-like (resonant) cascade will tend to dominate the decay of $W$ energy, and hence the decay of total energy also. It is our impression that this regime is closely related to the weak turbulence one. ${ }^{40,41}$ However, if $Z / W \ll W / V_{A}$, then the Shebalin-like cascade is weak compared to the IroshnikovKraichnan ("wave-wave") cascade, which will thus have primacy.

Finally, we note that extensions of the model are being developed. These will take into account nonzero crosshelicity effects, for example, and include forcing terms. Such extensions will improve the usefulness of the model, particularly for applications like pickup ion driving of solar wind turbulence. In that system, $Z$ appears to control the dissipation in the inner heliosphere, but in the outer heliosphere pickup ions are most likely injecting $W$ excitations. ${ }^{43-45}$

\section{ACKNOWLEDGMENTS}

This research was supported by grants from the NSF (Grant No. ATM-0539995), NASA (Grant No. NNG04GA54G), and the NZ Marsden Fund (Grant No. 02UOW-050 MIS).

${ }^{1}$ G. I. Taylor, Proc. R. Soc. London, Ser. A 151, 421 (1935).

${ }^{2}$ T. von Kármán and L. Howarth, Proc. R. Soc. London, Ser. A 164, 192 (1938).

${ }^{3}$ H. L. Dryden, Q. Appl. Math. 1, 7 (1943).

${ }^{4} \mathrm{G}$. K. Batchelor, The Theory of Homogeneous Turbulence (Cambridge University Press, Cambridge, 1970).

${ }^{5}$ A. N. Kolmogorov, C. R. Acad. Sci. URSS 32, 16 (1941) [reprinted in Proc. R. Soc. London, Ser. A 434, 15 (1991)].

${ }^{6}$ M. Hossain, P. C. Gray, D. H. Pontius Jr., W. H. Matthaeus, and S. Oughton, Phys. Fluids 7, 2886 (1995).

${ }^{7}$ D. Biskamp and W.-C. Müller, Phys. Plasmas 7, 4889 (2000).

${ }^{8}$ W.-C. Müller and D. Biskamp, in Turbulence and Magnetic Fields in 
Astrophysics, edited by E. Falgarone and T. Passot, Vol. 614 of Lecture Notes in Physics (Springer, 2003), pp. 3-26.

${ }^{9}$ W. H. Matthaeus, S. Oughton, D. Pontius, and Y. Zhou, J. Geophys. Res. 99, 19267 (1994).

${ }^{10}$ D. C. Montgomery and L. Turner, Phys. Fluids 24, 825 (1981).

${ }^{11}$ G. P. Zank and W. H. Matthaeus, Phys. Fluids A 5, 257 (1993).

${ }^{12}$ A. Pouquet, U. Frisch, and J. Léorat, J. Fluid Mech. 77, 321 (1976)

${ }^{13}$ J. A. Domaradzki and R. S. Rogallo, Phys. Fluids A 2, 413 (1990).

${ }^{14}$ G. L. Eyink, Physica D 207, 91 (2005).

${ }^{15}$ M. K. Verma, A. Ayyer, and A. V. Chandra, Phys. Plasmas 12, 082307 (2005).

${ }^{16}$ W. H. Matthaeus, M. L. Goldstein, and D. A. Roberts, J. Geophys. Res. 95, 20673 (1990).

${ }^{17}$ W. H. Matthaeus, S. Oughton, S. Ghosh, and M. Hossain, Phys. Rev. Lett. 81, 2056 (1998).

${ }^{18}$ P. Goldreich and S. Sridhar, Astrophys. J. 438, 763 (1995).

${ }^{19}$ R. Kinney and J. C. McWilliams, Phys. Rev. E 57, 7111 (1998).

${ }^{20}$ S. Oughton, S. Ghosh, and W. H. Matthaeus, Phys. Plasmas 5, 4235 (1998).

${ }^{21}$ S. Oughton, P. Dmitruk, and W. H. Matthaeus, Phys. Plasmas 11, 2214 (2004).

${ }^{22}$ J. W. Bieber, W. Wanner, and W. H. Matthaeus, J. Geophys. Res. 101, 2511 (1996).

${ }^{23}$ C.-Y. Tu and E. Marsch, J. Geophys. Res. 98, 1257 (1993).

${ }^{24}$ J. W. Bieber, W. H. Matthaeus, C. W. Smith, W. Wanner, M. Kallenrode, and G. Wibberenz, Astrophys. J. 420, 294 (1994).

${ }^{25}$ M. Dobrowolny, A. Mangeney, and P. Veltri, Phys. Rev. Lett. 45, 144 (1980).

${ }^{26}$ R. Grappin, U. Frisch, J. Léorat, and A. Pouquet, Astron. Astrophys. 105, 6 (1982)
${ }^{27}$ W. H. Matthaeus, G. P. Zank, and S. Oughton, J. Plasma Phys. 56, 659 (1996).

${ }^{28}$ W.-C. Muller and D. Biskamp, Phys. Rev. Lett. 84, 475 (2000).

${ }^{29}$ R. Grappin, A. Pouquet, and J. Léorat, Astron. Astrophys. 126, 51 (1983).

${ }^{30}$ W. H. Matthaeus and Y. Zhou, Phys. Fluids B 1, 1929 (1989).

${ }^{31}$ R. H. Kraichnan, Phys. Fluids 8, 1385 (1965).

${ }^{32}$ W. D. McComb, The Physics of Fluid Turbulence (Oxford University Press, New York, 1990).

${ }^{33}$ M. Lesieur, Turbulence in Fluids, 2nd ed. (Kluwer, Dordrecht, The Netherlands, 1990).

${ }^{34}$ P. S. Iroshnikov, Astron. Zh. 40, 742 (1963) [Sov. Astron. 7, 566 (1964)].

${ }^{35}$ J. V. Shebalin, W. H. Matthaeus, and D. Montgomery, J. Plasma Phys. 29, 525 (1983).

${ }^{36}$ S. Oughton, E. R. Priest, and W. H. Matthaeus, J. Fluid Mech. 280, 95 (1994).

${ }^{37}$ G. P. Zank, W. H. Matthaeus, and C. W. Smith, J. Geophys. Res. 101, 17093 (1996).

${ }^{38}$ A. Bondeson, Phys. Fluids 28, 2406 (1985).

${ }^{39}$ D. Biskamp and E. Schwarz, Phys. Plasmas 8, 3282 (2001).

${ }^{40}$ S. Galtier, S. V. Nazarenko, A. C. Newell, and A. Pouquet, J. Plasma Phys. 63, 447 (2000).

${ }^{41}$ S. Galtier, S. V. Nazarenko, A. C. Newell, and A. Pouquet, Astrophys. J. Lett. 564, L49 (2002).

${ }^{42}$ A. N. Kolmogorov, Dokl. Akad. Nauk SSSR 30, 301 (1941) [reprinted in Proc. R. Soc. London, Ser. A 434, 9 (1991)].

${ }^{43}$ W. H. Matthaeus, G. P. Zank, C. W. Smith, and S. Oughton, Phys. Rev. Lett. 82, 3444 (1999).

${ }^{44}$ P. A. Isenberg, C. W. Smith, and W. H. Matthaeus, Astrophys. J. 592, 564 (2003).

${ }^{45}$ P. A. Isenberg, Astrophys. J. 623, 502 (2005). 